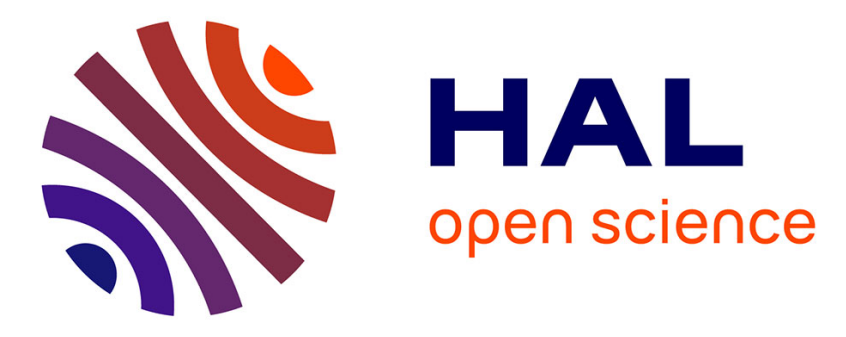

\title{
Uncertainty component estimates in transient climate projections.
}

Benoit Hingray, Juliette Blanchet, Guillaume Evin, Jean-Philippe Vidal

\section{To cite this version:}

Benoit Hingray, Juliette Blanchet, Guillaume Evin, Jean-Philippe Vidal. Uncertainty component estimates in transient climate projections.: Precision of estimators in a single time or time series approach. Climate Dynamics, 2019, 53 (5-6), pp.2501-2516. 10.1007/s00382-019-04635-1 . hal02327452

\section{HAL Id: hal-02327452 \\ https://hal.science/hal-02327452}

Submitted on 26 Nov 2020

HAL is a multi-disciplinary open access archive for the deposit and dissemination of scientific research documents, whether they are published or not. The documents may come from teaching and research institutions in France or abroad, or from public or private research centers.
L'archive ouverte pluridisciplinaire HAL, est destinée au dépôt et à la diffusion de documents scientifiques de niveau recherche, publiés ou non, émanant des établissements d'enseignement et de recherche français ou étrangers, des laboratoires publics ou privés. 
Noname manuscript No.

(will be inserted by the editor)

\title{
1 Uncertainty component estimates in transient climate projections \\ Precision of estimators in a single time or time series approach
}

\author{
Benoit Hingray - Juliette Blanchet . \\ Guillaume Evin · Jean-Philippe Vidal \\ Received: date / Accepted: date
}

\begin{abstract}
Quantifying model uncertainty and internal variability components in climate projections has been paid a great attention in the recent years. For multiple synthetic ensembles of climate projections, we compare the precision of uncertainty component estimates obtained respectively with the two Analysis of Variance (ANOVA) approaches mostly used in recent works: the popular Single Time approach (STANOVA), based on the data available for the considered projection lead time and a time series based approach (QEANOVA), which assumes quasi-ergodicity of climate outputs over the available simulation period.

18 We show that the precision of all uncertainty estimates is higher when more members are used, when internal variability is smaller and/or the response-touncertainty ratio is higher. QEANOVA estimates are much more precise than STANOVA ones: QEANOVA simulated confidence intervals are roughly 3 to 5 times smaller than STANOVA ones. Except for STANOVA when less than 3 members is available, the precision is rather high for total uncertainty and moderate for internal variability estimates. For model uncertainty or responseto-uncertainty ratio estimates, the precision is low for QEANOVA to very low for STANOVA. In the most unfavorable configurations (small number of members, large internal variability), large over- or underestimation of uncertainty components is thus very likely. In a number of cases, the uncertainty analysis 29 should thus be preferentially carried out with a time series approach or with a
\end{abstract}

B. Hingray

Univ. Grenoble Alpes, CNRS, IGE UMR 5001, Grenoble, F-38000, France

E-mail: benoit.hingray@ujf-grenoble.fr

J. Blanchet

Univ. Grenoble Alpes, CNRS, IGE UMR 5001, Grenoble, F-38000, France

G. Evin

Univ. Grenoble Alpes, Irstea, UR ETNA, Grenoble, F-38000, France

J.-P. Vidal

Irstea, UR RiverLy, centre de Lyon-Villeurbanne, F-69625 Villeurbanne, France 
local-time series approach, applied to all predictions available in the temporal neighborhood of the target prediction lead time.

Keywords Uncertainty Sources · Climate Projections · ANOVA · Internal Variability · Model Uncertainty · Scenario Uncertainty · Precision of Estimates

\section{Introduction}

A critical issue in climate change studies is the estimation of uncertainties in projections along with the contribution of the different uncertainty sources, including scenario uncertainty, the different components of model uncertainty, and internal variability (e.g. Hawkins and Sutton, 2009).

Scenario uncertainty is related to the poorly known future of greenhouse gas emissions. Model uncertainty corresponds to the dispersion between the different climate responses obtained with different models for the same forcing configuration. Model uncertainty concerns Global Climate Models (GCMs) but also all subsequent models of the climate change impact modeling chain, such as Regional Downscaling Models (regional climate models and/or statistical downscaling methods) and impact models like Hydrological Models (HMs). Internal variability first originates from the chaotic variability of the climate at the global scale (e.g. Räisänen, 2001; Deser et al, 2012). At the local scale, it also results from the fact that very similar large scale atmospheric circulation configurations can lead to very different meteorological observations (Braun et al, 2012; Lafaysse et al, 2014).

Estimating and partitioning uncertainty in future climate projections is first intended to help evaluating the significance of estimated changes for adaptation purposes. Besides, this is intended to highlight the most important uncertainty sources. This thus allows estimating the fraction of total uncertainty that could be narrowed via scenario refinement and model improvement, and its irreducible fraction pertaining to natural variability (e.g. Hawkins and Sutton, 2011; Lafaysse et al, 2014).

Over the recent years, uncertainty has been mostly explored and partitioned based on Multiscenarios Multimodel Multimember Ensembles (MMEs) of transient climate projections. Various methods have been proposed for this, most of them based on an Analysis of Variance (ANOVA) of projections available for the specific projection lead time considered (Hingray et al, 2007; Yip et al, 2011; Bosshard et al, 2013; Giuntoli et al, 2015; van Pelt et al, 2015; Paeth et al, 2017). In this single time approach - called STANOVA in the following -, and provided multiple members are available for each modeling chain, the model uncertainty components are estimated from the dispersion between the climate responses of the different modeling chains, obtained for each chain from the multimember mean of the projections. Similarly, the internal variability component for each modeling chain is estimated from the inter-member variance of the projections.

The different uncertainty components can also be estimated from a time series approach, benefiting from the long time series now available for most 
climate experiments (e.g. Johns et al, 2011; Jacob et al, 2014). A quasi-ergodic assumption for climate simulations in transient climate is often used in this context (Hingray and Saïd, 2014). It considers that the temporal variations of the climate response of a particular simulation chain are necessarily gradual and smooth, the higher frequency variations of the time series being due to internal variability alone. It considers next that the internal variability can be assumed to remain roughly constant over the considered period or to vary as a gradual and smooth function of the climate response of the chain. These assumptions were used for instance by Räisänen (2001); Hawkins and Sutton (2009, 2011); Charlton-Perez et al (2010); Lafaysse et al (2014); Bracegirdle et al (2014); Reintges et al (2017). In this time series approach called QEANOVA in the following, the climate change response of each simulation chain and its possible evolution with time is obtained from the long term trend estimated from the time series of the chain. The variance over time of the deviation from the climate response defines the internal variability of each chain. The climate responses of the different chains can be used to estimate the components of model uncertainty for any projection lead time, thanks again to a usual ANOVA.

In both approaches, a potentially critical step is the possibility to identify a precise estimate of the climate response of each modeling chain. A number of previous works advocated the use of multiple runs to improve this identification (e.g. Kendon et al, 2008; Deser et al, 2012; Kew et al, 2011). Unfortunately a limited number of members have until now been usually available for most modeling chains, logically deriving from the small number of GCM runs (e.g. Johns et al, 2011; Jacob et al, 2014; Paeth et al, 2017; Reintges et al, 2017). In a number of analyses, the discrimination between internal climate variability and the climate response of each modeling chain is thus expected to be quite inaccurate owing to the limited ensemble size especially when the internal variability is non-negligible compared to the chain's climate responses (e.g. Deser et al, 2012; van Pelt et al, 2015).

These inaccuracies translate to inaccuracies in estimates of uncertainty components and of derived characteristics (e.g. response to uncertainty ratio, significance of projected changes, time of emergence of climate change, etc.). In specific analysis configurations, the precision of uncertainty component estimates is potentially low to very low, but to our knowledge this issue has never been considered.

In the following, we characterize the precision of uncertainty components estimates when obtained either with the single time or the time series approaches introduced previously. The precision is obviously expected to depend on the multimodel multimember ensemble of projections (MME) available for the analysis and on the climate variable considered for the analysis. In the following, both ANOVA approaches are applied to multiple synthetic MMEs obtained via Monte Carlo simulations. We consider a simplified configuration where MME account for two uncertainty sources: model uncertainty and internal variability. The analysis framework we follow here is rather crude but it allows comparing both ANOVA approaches for a large number of MME config- 
urations with different characteristics. We especially discuss how the precision depends on the number of members used in the analysis, and we characterize the gain in precision obtained with the time series based approach, owing to the larger size of the data set accounted for. We also discuss the results for different levels of internal climate variability contribution to total uncertainty variance. We first focus on the precision of estimates obtained for model uncertainty and internal variability. We next consider the precision of estimates obtained on the one hand for the mean climate response from the model ensemble and on the other hand for the response to uncertainty ratio frequently used to estimate the significance of estimated changes.

All recent analyses of uncertainty sources in climate projections make use of the moment estimators of variance components. The present study also makes use of such estimators in order to put the precision of uncertainty estimates derived from these analyses into perspective.

The statistical bases for the estimation of uncertainty components in a given MME are summarized in section 2 for both the single lead time ANOVA and the quasi-ergodic ANOVA approaches. The Monte Carlo experiment for the simulation of multiple synthetic MMEs is presented in section 3. The precision of uncertainty estimates is presented in section 4, and discussed in section 5. Section 6 concludes.

\section{ANOVA methods}

\subsection{Transient ensembles of climate experiments}

Let us consider a hypothetical ensemble of climate experiments where $M$ different members are available for each of $G$ different climate modeling chains. For the sake of simplicity, we assume that the same number of members is available for each chain. A chain refers for instance to a given GCM and the members to the different runs available for each GCM. A chain could also refer to a given GCM/RDM combination or to a given GCM/RDM/HM model combination. Members would respectively refer to the potentially multiple realizations obtained with the different runs for each GCM/RDM combination or for each GCM/RDM/HM combination (e.g. Lafaysse et al, 2014; Vidal et al, 2016).

Note $Y(g, m, t)$ the climate projection obtained for the $m^{\text {th }}$ member of chain $g$ for time $t$. $Y(g, m, t)$ can be expressed as:

$$
Y(g, m, t)=\lambda(g, t)+\nu(g, m, t),
$$

where $\lambda(g, t)$ is the climate response of model $g$ for year $t$ and $\nu(g, m, t)$ is the deviation from the climate response obtained with the member $m$ for this year as a result of internal variability (IV).

As in most climate impact studies, the uncertainty analysis can be carried out for the change variable $X$. In the following, we consider absolute changes, 
but a similar analysis could be obtained for relative changes. Absolute changes are expressed as:

$$
X(g, m, t)=Y(g, m, t)-Y\left(g, m, t_{C}\right),
$$

where $X(g, m, t)$ are the experiment outputs of the change variable for a given member $m$ of each chain $g$, each year $t$ and where $t_{C}$ refers to a given reference year (or $n$-yr reference period centered on year $t_{C}$ ). These change outputs can be written as:

$$
X(g, m, t)=\varphi(g, t)+\eta(g, m, t),
$$

where $\varphi(g, t)$ is the climate change response of chain $g$ for $t$ and where $\eta(g, m, t)$ are the residuals obtained for the $m^{\text {th }}$ member of chain $g$ as a result of IV. In the following, we assume that the climate change response can be further decomposed as:

$$
\varphi(g, t)=\mu(t)+\alpha(g, t),
$$

where $\mu(t)$ is the mean climate change response from all available chains at projection lead time $t, \alpha(g, t)$ is the deviation from $\mu(t)$ for the climate change response of chain $g$.

By assumption, no correlation is expected between the change responses and the residuals $\eta^{\prime} s$. If the residuals are additionally independent and identically distributed (i.i.d.), the total uncertainty of the change variable at $t$ is $\sigma_{X}^{2}(t)=s_{\alpha}^{2}(t)+\sigma_{\eta}^{2}(t)$ where $s_{\alpha}^{2}(t)$ is the sample variance of the $\alpha$ 's in equation (4) and $\sigma_{\eta}^{2}(t)$ the variance of the $\eta^{\prime} s$ in equation (3). They respectively correspond to the model uncertainty and IV components of total uncertainty variance for $X$. Note that, for any given chain $g$, no correlation is expected between the values of the residuals $\nu(g, m, t)$ obtained from the raw variable $Y$ in the reference and in a future climate. The IV of the change variable $X$ is thus the sum of the IV estimates for $Y$ obtained for the reference and the future periods respectively.

In the following, we detail the two ANOVA approaches used to estimate (1) the mean climate change response $\mu(t),(2)$ the deviations $\alpha(g, t)$ for the different models, (3) the total uncertainty variance $\sigma_{X}^{2}(t),(4)$ its model uncertainty $s_{\alpha}^{2}(t)$, and (5) its IV component $\sigma_{\eta}^{2}(t)$. We will also consider the Response-toUncertainty ratio $\mathrm{R} 2 \mathrm{U}(t)$ defined as $\mathrm{R} 2 \mathrm{U}(t)=\mu(t) / \sigma_{X}(t)$, the fraction $F_{\alpha}(t)$ of total variance explained by model uncertainty and the fraction $F_{\eta}(t)$ of total variance explained by internal variability. Moment estimators of variance components are used here for both ANOVA approaches.

\subsection{Single Time ANOVA method}

The Single Time ANOVA method (STANOVA in the following) is by far the most frequently used approach for the last years. It consists in estimating an ANOVA model on the projected changes $X(g, m, t)$ for each time step $t$ in turn. For any given $t$, climate projections data available for the time steps that precede and follow $t$ are disregarded. 
The STANOVA method uses an ANOVA model with fixed effects of the form of equations (3) and (4). The residuals are assumed to be i.i.d. In this configuration, an unbiased estimator of the residual's variance $\sigma_{\eta}^{2}$ at time $t$ is the multimodel mean of the inter-member variance at $t$, expressed as (Montgomery, 2012):

$$
\widehat{\sigma}_{\eta, S T}^{2}(t)=\frac{1}{G(M-1)} \sum_{g=1}^{G} \sum_{m=1}^{M}\left\{X(g, m, t)-\widehat{\varphi}_{S T}(g, t)\right\}^{2}
$$

where $\widehat{\varphi}_{S T}(g, t)$ is an estimate of the mean climate change response for model $g$ at $t$ estimated over all $M$ members as $\widehat{\varphi}_{S T}(g, t)=\frac{1}{M} \sum_{m=1}^{M} X(g, m, t)$.

The model uncertainty variance is the inter-model sample variance of the deviations $\alpha(g, t)$ for all $g$, namely $s_{\alpha}^{2}(t)=\frac{1}{G-1} \sum_{g=1}^{G}\{\alpha(g, t)\}^{2}$. An unbiased estimator of $s_{\alpha}^{2}(t)$ under constraint $\sum_{g=1}^{G} \widehat{\alpha}_{S T}(g, t)=0$ is (Montgomery, 2012):

$$
\widehat{s}_{\alpha, S T}^{2}(t)=\frac{1}{G-1} \sum_{g=1}^{G}\left\{\widehat{\alpha}_{S T}(g, t)\right\}^{2}-\frac{1}{M} \widehat{\sigma}_{\eta, S T}^{2}(t)
$$

where $\widehat{\alpha}_{S T}(g, t)$ is an estimate of the deviation $\alpha(g, t)$ for model $g$, namely $\widehat{\alpha}_{S T}(g, t)=\widehat{\varphi}_{S T}(g, t)-\widehat{\mu}_{S T}(t)$ with $\widehat{\mu}_{S T}(t)$ the overall mean climate change response estimate at $t$, namely $\widehat{\mu}_{S T}(t)=\frac{1}{G} \sum_{g=1}^{G} \widehat{\varphi}_{S T}(g, t)$.

An unbiased estimator of the total variance for $X(g, m, t)$ finally reads: $\widehat{\sigma}_{X, S T}^{2}(t)=\widehat{s}_{\alpha, S T}^{2}(t)+\widehat{\sigma}_{\eta, S T}^{2}(t)$.

\subsection{Quasi-Ergodic ANOVA method}

A time series ANOVA approach consists first in estimating for each chain $g$ the climate response from the raw projections $Y(g, m, t)$. This is typically achieved by fitting a trend model to one of the members available for each chain (e.g. Hawkins and Sutton, 2009; Reintges et al, 2017). In the Quasi-Ergodic ANOVA method (QEANOVA in the following) considered here, the trend model is not obtained from one given member but from all the $M$ members $Y(g, m, t)$ available for the chain (Hingray and Saï, 2014). The trend model is assumed to give an unbiased estimator of the climate response function $\lambda(g, t)$ of the chain (the limitations linked to the choice of the trend model are discussed in section 5.2). In this case, the trend model for $Y$ gives an unbiased estimator of the climate change response function of the chain $\varphi(g, t)$, as:

$$
\widehat{\varphi}_{Q E}(g, t)=\widehat{\lambda}_{Q E}(g, t)-\widehat{\lambda}_{Q E}\left(g, t_{C}\right)
$$

where $\widehat{\lambda}_{Q E}(g, t)$ and $\widehat{\lambda}_{Q E}\left(g, t_{C}\right)$ are the trend estimates of the raw projections $Y$ for the future and reference periods respectively.

Under the quasi-ergodic assumption, the IV of chain $g$ for the raw data $Y$ is estimated as the variance over time of the residuals from the climate response estimated for the chain, namely $\left\{Y(g, m, t)-\hat{\lambda}_{Q E}(g, t)\right\}$. Note that all residuals 
from all members are used here. If the experiment period covers $T$ time steps, the IV of chain $g$ is thus estimated from a sample of size $T \times M$. Assuming no correlation between residuals obtained for the reference and future periods and assuming that the IV variance for $Y$ is constant over the entire experiment period, the IV of chain $g$ for the change variable $X$ is twice that of the raw variable $Y$ (Hingray and Saïd, 2014).

An unbiased estimator of the IV component for $X$ is derived from the multimodel mean of the IV estimates obtained for the $G$ different chains respectively (Hingray and Blanchet, 2018, see also Appendix A, Eq.A.3 and Eq.A.6). When for each chain $g$, the trend model for $Y$ can be expressed as a linear combination of $L$ functions of time, it reads :

$$
\widehat{\sigma}_{\eta, Q E}^{2}=\frac{2}{G(T M-L)} \sum_{g=1}^{G} \sum_{m=1}^{M} \sum_{k=1}^{T}\left\{Y\left(g, m, t_{k}\right)-\hat{\lambda}_{Q E}\left(g, t_{k}\right)\right\}^{2} .
$$

When the trend model is a simple linear function of time, $L=2$.

An unbiased estimator of the sample model uncertainty variance $s_{\alpha}^{2}(t)$ under constraint $\sum_{g=1}^{G} \widehat{\alpha}_{Q E}(g, t)=0$ is (Hingray and Blanchet, 2018, see also Appendix A, Eq.A.5):

$$
\widehat{s}_{\alpha, Q E}^{2}(t)=\frac{1}{G-1} \sum_{g=1}^{G}\left\{\widehat{\alpha}_{Q E}(g, t)\right\}^{2}-\frac{A(t, \mathcal{C})}{M} \widehat{\sigma}_{\eta, Q E}^{2},
$$

where $\widehat{\alpha}_{Q E}(g, t)$ is as previously an estimate of the deviation $\alpha(g, t)$ for model $g$ expressed as $\widehat{\alpha}_{Q E}(g, t)=\widehat{\varphi}_{Q E}(g, t)-\widehat{\mu}_{Q E}(t)$ with $\widehat{\mu}_{Q E}(t)=\frac{1}{G} \sum_{g=1}^{G} \widehat{\varphi}_{Q E}(g, t)$ the overall mean climate change response at $t . A(t, \mathcal{C})$ depends on the projection lead time and on the different functions of time used for the trend model of $Y$ (Hingray and Blanchet, 2018). It is positive and smaller than 1. The expression of $A(t, \mathcal{C})$ is given in Appendix $\mathrm{A}$ when the trend model is a simple linear function of time (Eq. A.7).

An unbiased estimator of total variance for $X(g, m, t)$ finally also reads: $\widehat{\sigma}_{X, Q E}^{2}(t)=\widehat{s}_{\alpha, Q E}^{2}(t)+\widehat{\sigma}_{\eta, Q E}^{2}$.

\section{Simulations}

In the following, we mainly focus on the precision of uncertainty estimators obtained with STANOVA and QEANOVA methods for the change variable $X\left(g, m, t_{e}\right)$ obtained between some future prediction lead time $t_{e}$ and the reference period $t_{C}$.

We construct via Monte Carlo simulations multiple synthetic MMEs where each MME is composed of $M \times G$ times series of raw projections $Y(g, m, t)$, with $M$ the number of members for each of the $G$ chains (see Appendix $\mathrm{C}$ for details). For reasons of simplification, each MME is simulated based on the assumptions that the climate response function of each modeling chain $g$ 
is a linear function of time and that the random deviations $\nu(g, m, t)$ due to internal variability come from independent and normally distributed random variables: $\nu(g, m, t) \sim \mathcal{N}\left(0, \sigma_{\nu}^{2}\right)$.

Each MME simulated for $Y(g, m, t)$ allows deriving the corresponding time series for $X(g, m, t)$. In the present analysis, for graphical simplification purposes, each MME for $Y$ is constructed so that it leads, for $X$ at time $t=t_{e}$, to a prescribed value of the Response-to-Uncertainty ratio $\left[\mathrm{R} 2 \mathrm{U}\left(t_{e}\right)\right]$ and to a prescribed value of the fractional variance $F_{\eta}\left(t_{e}\right)$ due to internal variability (Appendix C).

In the following, simulations were produced over the 1960-2100 period for a 10-yr average climate variable (see illustrative MMEs in figure 1). Decadal time series were next aggregated to 20 -yr time series for all analyses presented in section 4 , in order to match with the usually preferred 20-yr temporal resolution in climate analyses. $Y(g, m, t)$ time series are thus constituted from 7 values each $(T=140 / 20)$. The reference period is $t_{C}=1990$.

For the sake of simplicity, a unique number of chains is considered in the following section $(G=5)$. The influence of the number of chains on the results is discussed in section 5.1. Most results are finally presented for prediction lead time $t_{e}=2050$. $t_{e}$ will be thus omitted in most following notations.

\section{[ FIGURE 1 HERE ]}

For each MME, both STANOVA and QEANOVA models are applied, allowing estimating the ratio $\mathrm{R}(\theta)=\widehat{\theta} / \theta_{t h}$ between the estimated value $\widehat{\theta}$ and the theoretical (prescribed) value $\theta_{t h}$ of different parameters $\theta$ : the grand ensemble mean response $\mu\left(t_{E}\right)$, the uncertainty variances $s_{\alpha}^{2}\left(t_{E}\right), \sigma_{\eta}^{2}\left(t_{E}\right), \sigma_{X}^{2}\left(t_{E}\right)$, the Response-to-Uncertainty ratio $\left[\mathrm{R} 2 \mathrm{U}\left(t_{E}\right)\right]$ and the contribution to total variance of internal variability and model uncertainty, $F_{\eta}\left(t_{E}\right)$ and $F_{\alpha}\left(t_{E}\right)$.

We run the simulations for different combinations of prescribed R2U and $F_{\eta}$ values and for different numbers of members $M$. For each combination, we simulate 1,000 MMEs. We subsequently estimate, for each parameter $\theta$, the mean $\mathrm{E}[\mathrm{R}(\theta)]$ and the standard deviation $\mathrm{SD}[\mathrm{R}(\theta)]$ of the corresponding 1,000 ratios $\mathrm{R}(\theta)$.

Application of a QEANOVA method requires fitting a trend model to the raw climate projections $Y$. A parametric model is usually used for convenience. For simplicity, we here also assume that the trend is a linear function of time for the whole transient period $\left[t_{1}, t_{T}\right]$ (the influence of the trend model on the results is discussed in section 5.2). The estimation is made using ordinary least squares.

Equations (5) and (8) give unbiased estimators of $\sigma_{\eta}^{2}$. Respectively, equations (6) and (9) give unbiased estimators of $s_{\alpha}^{2}$. $\mathrm{E}[\mathrm{R}(\theta)]$ obtained for $\sigma_{\eta}^{2}$ and $s_{\alpha}^{2}$ with both STANOVA and QEANOVA approaches are therefore expected to equal one whatever the $\left(M, F_{\eta}, \mathrm{R} 2 \mathrm{U}\right)$ configuration. This actually also applies to all other STANOVA and QEANOVA estimators (not shown).

We therefore focus on the precision of estimators from STANOVA and QEANOVA approaches, i.e. on results obtained for $\mathrm{SD}[\mathrm{R}(\theta)]$, denoted SD below for shortness. The smaller the standard deviation, the better the precision of 
the estimator. We will additionally look at the ratio $S D_{S T} / S D_{Q E}$, denoting the gain in precision obtained with the QEANOVA approach with respect to the STANOVA approach. The larger this ratio, the larger the gain.

The uncertainty analysis with the STANOVA method requires at least two members for each chain. This is not the case for the QEANOVA method which can be carried out with no more than one single member for each chain. In the following, we also present, for information, results obtained with the QEANOVA method in case of single member MMEs.

\section{Results}

In our case and whatever the $\left(M, F_{\eta}, \mathrm{R} 2 \mathrm{U}\right)$ configuration, the distribution of the $R(\theta)$ ratios obtained respectively from the 1000 synthetic data sets was always found to be roughly normal (not shown). In a first approximation, the SD value of $R(\theta)$ thus determines the probability that the $R(\theta)$ ratio is outside of any $[1 \pm e]$ interval, that is the probability that the estimated value of the considered parameter is at least $100 e \%$ smaller or $100 e \%$ greater than the theoretical value. For instance, the probability that the ratio $\mathrm{R}(\theta)$ is outside the $[0.5,1.5]$ interval is $1 \%, 32 \%$ or $62 \%$ when SD is $0.2,0.5$ or 1 respectively. When the $\mathrm{SD}$ value is greater than 0.5 , the probability for a large underestimation (let say $\mathrm{R}(\theta)<0.5$ ) or for a large overestimation (let say $\mathrm{R}(\theta)>1.5$ ) is thus non negligible (i.e. $p>0.32$ ). When $\mathrm{SD}$ is greater than 1 , a large underestimation or overestimation of the considered parameter is very likely (i.e. $p>0.62$ ).

\subsection{Variance components, total and fractional variance}

SD results obtained for variance parameters $\left(\sigma_{\eta}^{2}, s_{\alpha}^{2}, \sigma_{X}^{2}, F_{\eta}, F_{\alpha}\right)$ are roughly independent on the $\mathrm{R} 2 \mathrm{U}$ value prescribed for the simulation of the synthetic MMEs (not shown). They conversely depend on the value prescribed for $F_{\eta}$ and on the number of members $M$. We here therefore only present results via the SD response functions in the $\left(M, F_{\eta}\right)$ domain.

For the different variance parameters and both ANOVA methods, SD values are logically higher for small $M$ numbers. Except for internal variability, they are also higher for large $F_{\eta}$ values, highlighting the lower precision of the estimators in these configurations (figure 2). For all parameters, STANOVA SD values are also logically higher than QEANOVA SD values.

\section{[ FIGURE 2 HERE ]}

For internal variability estimates, $\sigma_{\eta}^{2}$, SD values do only depend on $M$. For configurations with $M \leq 3$, STANOVA SD values are greater than 0.4, highlighting the possibility for significant errors in $\sigma_{\eta}^{2}$ estimates. Whatever the $\left(M, F_{\eta}\right)$ configuration, QEANOVA SD values are at least 3 times smaller than STANOVA SD values. QEANOVA estimates are thus expected to be much closer to the theoretical value. 
For model uncertainty estimates $s_{\alpha}^{2}$, the precision gain obtained with QEANOVA

is at least 2.5. It is up to 4 for high $F_{\eta}$ values (e.g. $>90 \%$ ) or even more when $M$ is smaller than 5 . Whatever the $\left(M, F_{\eta}\right)$ configuration, SD values are much larger than those obtained for internal variability. In the most unfavorable configurations (very low $M$ and very high $F_{\eta}$ values), STANOVA SD values are noticeably higher than $6 ! s_{\alpha}^{2}$ estimates are very likely to be largely over or underestimated. In those cases, a misestimation can also be obtained with QEANOVA but it would be somehow less critical.

Compared to $s_{\alpha}^{2}$ and $\sigma_{\eta}^{2}$, the potential error for total variance estimates, $\sigma_{X}^{2}$, is likely to be small for both methods. SD values are globally smaller than 0.2 for QEANOVA. For STANOVA, they are smaller than 0.3 except in the low $M$ and high $F_{\eta}$ configurations. This suggests that for any given dataset, errors obtained for $s_{\alpha}^{2}$ and $\sigma_{\eta}^{2}$ partly compensate. The gain in precision obtained with QEANOVA is around 2.5, whatever the $\left(M, F_{\eta}\right)$ configuration.

SD response functions obtained for fractional variances estimates, $F_{\eta}$ and $F_{\alpha}$, are very similar to those obtained for $\sigma_{\eta}^{2}$ and $s_{\alpha}^{2}$ estimates respectively (not shown). Whatever the method, errors in $F_{\eta}$ estimates are thus not expected to be very large. For $F_{\alpha}$, large errors are conversely expected with STANOVA in the most unfavorable $\left(M, F_{\eta}\right)$ configurations. The gain in precision obtained with QEANOVA is again at least 2.5.

\subsection{Mean response and Response-to-Uncertainty ratio}

We focus here on the estimates obtained for the mean change response $\mu$ and for the Response-to-Uncertainty ratio R2U. For both ANOVA methods and both parameters, SD values are again logically higher for smaller $M$ and/or higher $F_{\eta}$ values. SD values additionally depend on the theoretical value prescribed for the Response-to-Uncertainty ratio R2U. SD values decrease when the theoretical value for $\mathrm{R} 2 \mathrm{U}$ increases.

Results are presented in Figure 3 for $\mu$ estimates and three theoretical $\mathrm{R} 2 \mathrm{U}$ values $(0.2,1$, and 5$)$. They illustrate typical configurations encountered in climate analyses. A R2U value as high as 5 can be for instance typically obtained in the 2050s for large scale temperature variables (e.g. global mean temperature as in Hawkins and Sutton (2011)). A R2U value as low as 0.2 can be conversely obtained for noisy systems, e.g. regional climate variables with a high natural variability (e.g. 20-yr average regional precipitation as in Hingray and Saïd (2014)).

For $\mu$ estimates, STANOVA SD values obtained when $\mathrm{R} 2 \mathrm{U}=0.2$ are often greater than 0.5 and even exceed 2.5 for the most unfavorable $\left(M, F_{\eta}\right)$ configurations. When $\mathrm{R} 2 \mathrm{U}=1, \mathrm{SD}$ values are much smaller. They are lower than 0.15 except for the low $M$ and high $F_{\eta}$ configurations. For larger prescribed $\mathrm{R} 2 \mathrm{U}$ values, $\mathrm{SD}$ values tend to be negligible: they are always smaller than 0.03 when $\mathrm{R} 2 \mathrm{U}=5$. QEANOVA SD values present a similar dependence on $M, F_{\eta}$ and R2U. They are however again much smaller than STANOVA SD values (roughly 2.5 times smaller whatever the $M, F_{\eta}$ and R2U configuration). 


\section{[ FIGURE 3 HERE ]}

For R2U estimates, the structure of SD response functions are very similar to those obtained for $\mu$ estimates (not shown). SD values are however systematically larger (from $+20 \%$ for $\mathrm{R} 2 \mathrm{U}=0.1$ to more than $+100 \%$ for $\mathrm{R} 2 \mathrm{U}=5$ ). For noisy systems and the most unfavorable $\left(M, F_{\eta}\right)$ configurations, large errors are therefore expected with STANOVA. Errors are conversely expected to be low to moderate with QEANOVA estimates, which are at least 2.5 times more precise.

\section{Discussion}

5.1 Dependence to the setup and size of the projection ensemble

For the sake of simplicity, we considered that MMEs are composed of 5 modelling chains. This is much less than the number of chains currently available in recent CMIP MMEs. We also considered that the same number of members is available for all simulations chains. This is obviously never the case in real MMEs which are mostly unbalanced. In real MMEs, a large number of model combinations is also typically missing when the projections are produced with chains composed of different models (e.g. not all GCM/RCM combinations are available when projections are produced with different RCMs from different GCM outputs).

Whatever the ANOVA method, estimates of the different uncertainty components are expected to be more precise when more members are available, when the number of chains is larger and/or when the number of missing model combinations is smaller. In most of the cases however, a time series approach is still expected to produce more precise estimates of uncertainty components than a single time approach. This obviously results from the larger sample size used for the estimation which allows for a more precise estimate of the climate response and of the internal variability of each simulation chain.

As an illustration, the previous analysis was repeated with MMEs composed of 10 simulation chains instead of 5 (see Supplementary Material, Figure A1). As expected, the precision of all uncertainty components estimates is better with 10 chains (for both approaches) and QEANOVA estimates are more precise than STANOVA ones. In the present case, the gains in precision are actually roughly the same than those presented previously with 5 chains.

\subsection{The trend estimation issue in the QEANOVA approach}

In the present case and for convenience, time series of the synthetic MMEs are simulated based on the assumption that the climate response of each chain is a linear function of time. The QEANOVA trend function used to estimate the climate response of each chain is also assumed to be a linear function of time. 
In real datasets of projections, the climate response likely evolves in a more complex way, perhaps differently in different chains. In practice, the precise form of the climate response is not known, and for small ensembles and noisy systems it may be quite difficult to guess and/or fit. If the chosen trend model were incorrect for some chain, the response function estimate could be locally biased. Incorrect trend models could then lead to significant errors in the estimation of the local effects of the chains, and additional uncertainty would arise, particularly if internal variability is small. Because of this trend estimation issue, the results of a time series approach can thus be sub-optimal. The linear assumptions retained for practical reasons in Section 3, make the QEANOVA results likely too optimistic since the assumed trend model is the true one. Thus the precision gain with QEANOVA in Section 4 is likely to overestimate the gain to be obtained with this method for real MMEs.

For real MMEs, non-linear models can be considered for the estimation of the trend (e.g. 4rd order polynomial used in Hawkins and Sutton (2009) and in Hingray and Said (2014) for temperature changes) and the risk of choosing non relevant trend models can be reduced with dedicated statistical tests and a visual check of the fit (if any expert knowledge can be used for instance to assess the climatic relevance of the estimated trend).

The trend estimation issue in a time series ANOVA approach, which may be critical for some MME configurations, requires thus specific care. Notwithstanding, a time series approach is likely to present a significant advantage in many cases, even when the trend is not linear, especially when internal variability is large. For illustration, we considered the same set of MMEs as previously but, instead of fitting a linear trend for estimating the climate response of each simulation chain, we fitted a 3rd order polynomial function. Results are presented in Figure A2 of Supplementary Material. As expected, whatever the configuration in the $\left(M, F_{\eta}\right)$ domain, the precision of QEANOVA estimates is lower with the polynomial trend model, especially for noisy MMEs and for small ensembles. Indeed, with a polynomial trend, the degrees of freedom are larger and in case of noisy systems, the local trend is often dominated by internal variability which logically leads to a lower precision of the estimated climate response. Nevertheless, QEANOVA is still much more precise than STANOVA. Note that a more relevant experiment would have been to simulate synthetic MMEs with non linear trends for the true trends but such a simulation is unfortunately not convenient and would have required to make different arbitrary and non representative choices (e.g. shape and amplitude of non linearity to be retained for each chain). The experiment discussed here thus likely again underplays the trend estimation issue in real MMEs where the true trends are not linear. Fitting higher order polynomials when the true trend is linear is indeed expected to introduce little error especially when the sample is large (the fit essentially yields the linear trend), but, as mentioned previously, the opposite does not hold: fitting a linear trend when the true trend is non linear (e.g. quadratic or cubic) will always yield local biases which may be large. 
Finally, another possible limitation of a time series ANOVA approach is when internal variability is assumed to be constant over time. This assumption may be not really valid as suggested by the recent work of Olonscheck and Notz (2017). As presented by Hingray and Saïd (2014) or Bracegirdle et al (2014), this assumption is not necessarily required and can also be relaxed. The internal variability can for instance be assumed to vary gradually from one lead time to the other (Hingray and Saïd, 2014). It can also be estimated from the variance of the residuals within a moving temporal window centered on the targeted prediction lead time (Bracegirdle et al, 2014).

\subsection{Toward a local-QEANOVA approach}

The potential limitations discussed above could make the apparent higher attractiveness of a time series approach somehow reduced for some MMEs configurations (e.g. MMEs with complex trend shapes and low noise).

However, if difficult to exploit over the whole available simulation period, the QEANOVA principles can be at least used locally, in the temporal neighborhood $\Omega(t)$ of each time point $t$. In such a local QEANOVA approach, the climate response of any given chain $g$ can be obtained for instance from local linear regression, in which the trend is assumed to be smooth so that the climate response $\lambda(g, t)$ of the chain can be approximated by a straight line in the neighborhood of $t$ (Cleveland, 1979). The residuals estimated from all data in $\Omega(t)$ can next be used to assess the local internal variability of the projections. When the uncertainty analysis is produced for the climate change variable, the local linear regression and the estimation of the local internal variability is done for both the reference time $t_{c}$ and the future lead time $t_{e}$. In this configuration, note that the internal variability no longer has to be assumed constant between both periods.

The theoretical developments of this local QEANOVA approach, given in Hingray and Blanchet (2018) are summarized in Appendix B. For illustration, the local QEANOVA approach was also applied to the different simulation ensembles considered in the previous sections. The neighborhood $\Omega(t)$ was here chosen to be composed from the 3 consecutive time steps centered around $t$. In this context, the risk that the linear trend is dominated by natural variability is likely important for noisy systems and small ensembles. This configuration would have led to a risk of underestimating the natural variability but this is not the case here thanks to the unbiased estimator derived for this variable (see Appendix B).

Results of the local QEANOVA are presented for internal variability, model uncertainty and for the Response-to-Uncertainty ratio in figure 4. Results are not a surprise. For all uncertainty parameters, the precision obtained with the local QEANOVA is significantly higher than that obtained with STANOVA (confidence intervals are 2 to 3 times smaller for the local QEANOVA). The precision of the local QEANOVA is conversely smaller than that obtained with 
QEANOVA. These results again logically derive from the different sizes of the samples used by the three methods.

A local QEANOVA is thus a promising alternative to STANOVA. The risk for a large misestimation of uncertainty components is indeed much lower. Despite its smaller precision, a local QEANOVA can be also a good alternative to a time series approach. When the time series of the simulation chain presents a low to moderate noise but has a complex and nonlinear climate response for instance, a local QEANOVA actually reduces the risk of having a biased estimate of the climate response, which may occur in a time series approach because of the choice of an inappropriate trend function (see section 5.2).

\section{[ FIGURE 4 HERE ]}

\subsection{Temporal variations of uncertainty component estimates}

Whatever the estimation method, the uncertainty estimates are typically derived for the different prediction lead times of a given climate simulation period. The temporal behavior of these estimates is worth considering as it may be a relevant indicator of their precision. In most cases, the climate response of each simulation chain is indeed expected to evolve gradually with time. In most cases, the different uncertainty components of a given MME are thus also expected to evolve gradually with time.

Similarly, uncertainty components for model uncertainty and scenario uncertainty should start from zero when the climate variable considered for the analysis is a climate change variable. The climate change response of any simulation chain is indeed expected to start from zero when the prediction lead time corresponds to the control period.

Large temporal fluctuations of uncertainty estimates and/or large values of model uncertainty for the control period should warn for the possible lack of robustness of the analysis. The possibility to consider the temporal variations of uncertainty estimates to assess their precision is illustrated for three synthetic data sets in Figure 5 for $s_{\alpha}^{2}$ and $\sigma_{\eta}^{2}$ and in Figure 6 for $F_{\eta}$. The three MMEs have been simulated following the simulation process described in section 3. Confidence intervals (colored bounds) of uncertainty estimates (white lines), obtained from Monte Carlo simulations similar to those carried out in section 4, highlight the much lower precision of STANOVA, even when the fractional variance due to internal variability is low. This is also clear from the temporal variations of uncertainty estimates. When only 3 members are available, the variations of STANOVA estimates are erratic and non-negligible whatever the value of $F_{\eta}$. They are much smoother with a local QEANOVA and they completely vanish with QEANOVA. As expected also, the model uncertainty component estimated with QEANOVA starts from zero at the beginning of the century and then gradually increases. This is thus fully coherent with the expected behavior of this uncertainty component (limit to zero for the control period). This is not the case for the two other methods, especially 
for STANOVA in the case of small ensembles and noisy systems (e.g. significant non zero values of model uncertainty can be achieved at time $t=2000$ in Figure 5, STANOVA, 2nd line).

\section{[ FIGURE 5 HERE ]}

$$
\text { [ FIGURE } 6 \text { HERE ] }
$$

\section{Conclusion}

6.1 Precision of different methods for partitioning uncertainty

Two ANOVA approaches are currently used for partitioning model uncertainty and internal variability components in ensembles of climate projections: the popular Single Time approach (STANOVA), based on the data available for the considered projection lead time and time series approaches such as the QEANOVA approach considered here. For multiple synthetic ensembles, we compared the precision of uncertainty component estimates obtained with both approaches. The main findings are as follows:

- In both cases, the precision is logically lower for noisy MMEs and/or small ensembles of members. The precision of the mean climate response and of the Response-to-Uncertainty ratio estimates are additionally lower when the theoretical (prescribed) Response-to-Uncertainty ratio is lower.

- In many configurations, QEANOVA estimates are expected to be more precise than STANOVA ones. This does not depend on the setup and size of the MME considered (unbalanced MMEs, MMEs with missing chains, MMEs with more GCMs,...). The larger sample size used for the estimation is indeed expected to produce a better estimate of the climate response and of the internal variability of each simulation chain.

- Whatever the estimation method, the temporal variations of the uncertainty estimates should be considered as a relevant indicator of the precision of estimates. Large temporal fluctuations should call for caution and should warn for the likely low relevance of the uncertainty analysis.

Under the specific setup and assumptions of our analysis framework (e.g. length of time series, number of simulations chains, linearity of trend, constancy of internal variability over time...), we find that:

- Simulated confidence intervals obtained with QEANOVA are at least 2.5 times smaller than those obtained with STANOVA.

- The precision of estimates is rather high for total uncertainty variance and for the multichain mean response (except for STANOVA for noisy MMEs and/or for MMEs with small ensembles). The two main features of any given ensemble of projections are thus likely to be rather correctly estimated in most cases. 
- This is obviously not the case for the individual uncertainty components. For model uncertainty variance for instance, the precision of estimates is low to very low for small ensembles and/or noisy MMEs. In the latter configurations, a large over- or underestimation of uncertainty components is thus very likely preventing any precise partition of uncertainty sources. This problem is particularly critical for STANOVA.

- STANOVA actually appears to be inappropriate in a large number of configurations as a result of the limited number of members, e.g. GCM runs, usually available for most modeling chains up to now. In the case of small ensembles and noisy MMEs, improving the relevance of uncertainty estimates requires using more data. A time series analysis approach as the QEANOVA method offers such an opportunity.

For a time series approach, the trend estimation can be an issue. For MMEs where internal variability is small for instance, there is some risk to misrepresent the forced trend. In such a case, a local QEANOVA approach offers an interesting alternative as it is not subject to the same limitation as a full time series approach. The precision of a local QEANOVA is additionally always better than that of STANOVA. Note also that, similarly to a time series approach, a local QEANOVA can be also applied when only a single member is available for the chains. It can therefore make use of all climate experiments produced in the recent years. This is another advantage over STANOVA which requires multiple members of each chain.

\subsection{Precision of uncertainty estimates for real ensembles of projections}

Most ensembles of projections have potentially more uncertainty sources than those considered in the present work. They may actually include scenario uncertainty, different components of model uncertainty (e.g. associated respectively to GCMs, Hydrological Models), of internal variability (with potentially its large or small scale components) and, eventually, uncertainty associated to interactions between models and/or scenarios (e.g. Hawkins and Sutton, 2011; Vidal et al, 2016; Paeth et al, 2017). In all cases, and whatever the ANOVA method used for the analysis, an idea of the precision of uncertainty components should be provided along with estimates of uncertainty components.

Results presented in the present work give already some benchmark for simple ensembles of projections. They were obtained with moment estimators of variance components. Likelihood-based estimation methods such as maximum likelihood, restricted maximum likelihood or Bayesian methods could (should) be used instead. They indeed allow for the estimation of confidence or credibility intervals associated to each uncertainty component. They also present next a number of interesting properties (e.g. Hartley and Rao, 1967; Gelman, 2005). Conversely to method-of-moment based ANOVA approaches, they can especially not produce negative estimates of variance components. For the two ANOVA methods considered here, negative estimates are indeed obtained for model uncertainty variance when the second terms in the right 
hand sides of equations (6) or (9) are greater than the first terms. The probability of having a negative estimate for $s_{\alpha}^{2}$ is actually directly related to the precision that can be achieved with the method for this component. For our work, this probability is roughly $2.5 \%, 16 \%$ or $31 \%$ when the SD value obtained for $s_{\alpha}^{2}$ is $0.5,1$ or 2 respectively. It can be up to $50 \%$ in the most critical $\left(M, F_{\eta}, \mathrm{R} 2 \mathrm{U}\right)$ configurations.

Likelihood-based estimation methods are also expected to use the data more efficiently than moment-based estimation methods. They are for instance easily applicable for unbalanced MMEs and/or MMEs with missing chains. The work of Northrop and Chandler (2014) and of Evin et al (2018) give some nice illustrations of their uses in a Bayesian context. The use of likelihoodbased estimation methods is thus to be preferred to the use of moment-based estimation methods. Even with such methods however, our results strongly suggest that a full- or partial time series approach should be used instead of a standard single time approach.

Author contributions. BH designed the analysis, developed the localQEANOVA and the synthetic simulations. JB derived the theoretical expressions for unbiased estimators of uncertainty components and wrote the appendixes. All authors contributed to write the manuscript and discuss results.

Acknowledgements We thank the three anonymous reviewers for their constructive suggestions which helped to significantly improve the content of our manuscript. 


\section{Appendix A QEANOVA Estimates of uncertainty components}

We summarize the theoretical developments of the QEANOVA approach to achieve unbiased estimators of uncertainty components for a simplified configuration where the trend model is a simple linear function of time. The full developments are given in Hingray and Blanchet (2018) for the general configuration where the trend model is a linear combination of $L$ functions of time. Here, we first consider the case where the number of members differs from one chain to the other; the simplified equations obtained when all chains have the same number are then given. For the sake of conciseness, we omit the subscript "QE" related to the QEANOVA approach.

Model. We first consider the raw projections $Y(g, m, t)$ with $M_{g}$ members for each of the $G$ chains, assuming that, for all $t_{s} \leq t \leq t_{f}$ :

$$
Y(g, m, t)=\lambda(g, t)+\nu(g, m, t),
$$

where $\lambda(g, t)$ is the trend model expressed as a linear function of time: $\lambda(g, t)=\Lambda_{g 1}+\Lambda_{g 2}\left(t-t_{s}\right)$ and where the $\nu(g, m, t)$ are independent and homoscedastic random variables (with variance $\sigma_{\nu_{g}}^{2}$ ). Second let consider the change variable at future prediction lead time $t \in\left[t_{c}, t_{f}\right]$ :

$$
X(g, m, t)=Y(g, m, t)-Y\left(g, m, t_{c}\right)=\alpha(g, t)+\eta(g, m, t),
$$

where $t_{c}$ is the reference period. We have thus $\alpha(g, t)=\Lambda_{g 2}\left(t-t_{c}\right)$ and $\eta(g, m, t)=\nu(g, m, t)-\nu\left(g, m, t_{c}\right)$.

Unbiased estimation of the model parameters for the raw variable $Y$. We discretize $\left[t_{s}, t_{f}\right]$ into $T$ time steps (from $t_{s}=t_{1}$ to $t_{f}=t_{T}$ ) and write $t_{c}$ as the $K$ th time step (i.e. $t_{c}=t_{K}$ ). We are interested in the future prediction lead time $t_{k} \in\left[t_{K}, t_{T}\right]$. Let consider the regression model (A.1) for a particular $g$. Unbiased estimators of the regression parameters $\left(\Lambda_{g 1}, \Lambda_{g 2}\right)$ are given by the least square estimates

$$
\left(\hat{\Lambda}_{g 1}, \hat{\Lambda}_{g 2}\right)^{\prime}=\mathbb{V} \mathbb{R}^{\prime}\left(\frac{1}{M_{g}} \sum_{m=1}^{M_{g}} Y\left(g, m, t_{1}\right), \ldots, \frac{1}{M_{g}} \sum_{m=1}^{M_{g}} Y\left(g, m, t_{T}\right)\right)^{\prime}
$$

where "' denotes the transpose, $\mathbb{R}$ is the $T \times 2$ matrix of covariates whose $k$ th row is $\left(1, t_{k}-t_{1}\right)$, for $1 \leq k \leq T$, and $\mathbb{V}=\left(\mathbb{R}^{\prime} \mathbb{R}\right)^{-1}$. Covariance matrix of the estimators $\left(\hat{\Lambda}_{g 1}, \hat{\Lambda}_{g 2}\right)$ is given by $\widehat{\sigma_{\nu_{g}}^{2}} M_{g}^{-1} \mathbb{V}$ where $\widehat{\sigma_{\nu_{g}}^{2}}$ is an unbiased estimator of $\sigma_{\nu_{g}}^{2}$ given by

$$
\widehat{\sigma_{\nu_{g}}^{2}}=\frac{1}{T M_{g}-L} \sum_{m=1}^{M_{g}} \sum_{k=1}^{T}\left\{Y\left(g, m, t_{k}\right)-\hat{\lambda}_{Q E}\left(g, t_{k}\right)\right\}^{2}
$$

where $L=2$ and $\hat{\lambda}_{Q E}\left(g, t_{k}\right)=\hat{\Lambda}_{g 1}-\hat{\Lambda}_{g 2}\left(t_{k}-t_{1}\right)$. 
In particular, an unbiased estimator of $\Lambda_{g 2}^{2}$ is

$$
\widehat{\Lambda_{g 2}^{2}}=\hat{\Lambda}_{g 2}^{2}-\widehat{\sigma_{\nu_{g}}^{2}} M_{g}^{-1} V_{22}
$$

where $V_{22}$ is the element $(2,2)$ of $\mathbb{V}$. Considering $t_{1}, \ldots, t_{T}$ regularly spaced on $\left[t_{s} ; t_{f}\right]$, we have $V_{22}=12(T-1) /\left\{T(T+1)\left(t_{T}-t_{1}\right)^{2}\right\}$.

Unbiased estimation of the sample variance of the $\alpha$ 's in the change variable. Given (A.2) and (A.4), an unbiased estimator of the sample variance of $\alpha\left(g, t_{k}\right)$, i.e. of $s_{\alpha}^{2}\left(t_{k}\right)=\frac{1}{G-1} \sum_{g=1}^{G}\left\{\alpha\left(g, t_{k}\right)\right\}^{2}$, is

$$
\widehat{s_{\alpha}^{2}}\left(t_{k}\right)=s_{\hat{\alpha}}^{2}\left(t_{k}\right)-\frac{12}{T} \frac{T-1}{T+1}\left(\frac{t_{k}-t_{K}}{t_{T}-t_{1}}\right)^{2}\left(\frac{1}{G} \sum_{g=1}^{G} \frac{\widehat{\sigma_{\nu_{g}}^{2}}}{M_{g}}\right),
$$

where

$$
s_{\hat{\alpha}}^{2}\left(t_{k}\right)=\frac{\left(t_{k}-t_{1}\right)^{2}}{G-1} \sum_{g=1}^{G} \hat{\Lambda}_{g 2}^{2} .
$$

When all GCMs have the same number of runs $(M)$, this expression reduces to:

$$
\widehat{s}_{\alpha}^{2}\left(t_{k}\right)=s_{\hat{\alpha}}^{2}\left(t_{k}\right)-\frac{A\left(t_{k}, \mathcal{C}\right)}{M} \widehat{\sigma}_{\eta}^{2},
$$

where $\widehat{\sigma}_{\eta}^{2}$ is an unbiased estimator of internal variability variance for $X$

$$
\widehat{\sigma}_{\eta}^{2}=\frac{2}{G} \sum_{g=1}^{G} \widehat{\sigma_{\nu_{g}}^{2}}
$$

and where

$$
A\left(t_{k}, \mathcal{C}\right)=\frac{6(T-1)}{T(T+1)}\left(\frac{t_{k}-t_{K}}{t_{T}-t_{1}}\right)^{2}
$$

\section{Appendix B Estimates with a local QEANOVA approach}

We here summarize the expressions of the different uncertainty estimators obtained with a local-QEANOVA approach. The full developments, similar to those presented in appendix A for the QEANOVA approach, are detailed in Hingray and Blanchet (2018).

Model. A regression model is still considered to estimate the response function $\lambda(g, t)$ for $Y$ in Eq.A.1 but $\lambda(g, t)$ is assumed to be only locally linear in time, in the neighborhoods of $t_{c}$ and $t_{e}$ respectively, i.e. on $\left[t_{c}-\omega, t_{c}+\omega\right]$ and $\left[t_{e}-\omega, t_{e}+\omega\right]$, where $t_{e} \in\left[t_{c}, t_{f}\right]$ is the future prediction lead time under consideration. $\lambda(g, t)$ can thus be expressed as

$$
\lambda(g, t)=\left\{\begin{array}{l}
\lambda_{c}(g, t)=\Lambda_{g 1, c}+\left(t-t_{c}\right) \Lambda_{g 2, c} \text { for } t_{c}-\omega \leq t \leq t_{c}+\omega, \\
\lambda_{e}(g, t)=\Lambda_{g 1, e}+\left(t-t_{e}\right) \Lambda_{g 2, e} \text { for } t_{e}-\omega \leq t \leq t_{e}+\omega .
\end{array}\right.
$$


The change variable $X(g, m, t)$, for $t_{e}-\omega \leq t \leq t_{e}+\omega$, in Eq.A.2 is such that $\alpha(g, t)=\left(\Lambda_{g 1, e}-\Lambda_{g 1, c}\right)+\left(t-t_{e}\right) \Lambda_{g 2, e}$.

Each interval $\left[t_{c}-\omega, t_{c}+\omega\right]$ and $\left[t_{e}-\omega, t_{e}+\omega\right]$ is discretized into $T^{\star}$ regular periods of length $d t=2 \omega /\left(T^{*}-1\right)$, with $T^{\star}$ odd, giving respectively the sequences $t_{1}, \ldots, t_{T^{*}}$ and $t_{T^{*}+1}, \ldots, t_{2 T^{*}}$. The values of $Y$ for these different times are further considered to estimate the regression coefficients of the linear trend models in Eq.B.1. For the illustration given in section 5.3, $d t=\omega=$ $20 y r s$ and $T^{*}=3$.

Unbiased estimators of model uncertainty and internal variability variance. Following Hingray and Blanchet (2018), an unbiased estimator of model uncertainty variance, i.e. of the sample variance of $\alpha\left(g, t_{e}\right)$, is

$$
\widehat{s_{\alpha}^{2}}\left(t_{e}\right)=s_{\hat{\alpha}}^{2}\left(t_{e}\right)-\frac{4}{T}\left(\frac{1}{G} \sum_{g=1}^{G} \frac{\widehat{\sigma_{\nu_{g}}^{2}}}{M_{g}}\right)
$$

where $T=2 T^{*}$ is the total number of time steps considered in the analysis and where $\widehat{\sigma_{\nu_{g}}^{2}}$ is an unbiased estimator of $\sigma_{\nu_{g}}^{2}$ given by

$$
\begin{aligned}
\widehat{\sigma_{\nu_{g}}^{2}}= & \frac{1}{T M_{g}-4} \sum_{m=1}^{M_{g}}\left[\sum_{j=1}^{T^{*}}\left\{Y\left(g, m, t_{j}\right)-\hat{\lambda}_{c}\left(g, t_{j}\right)\right\}^{2}\right. \\
& \left.+\sum_{j=T^{*}+1}^{2 T^{*}}\left\{Y\left(g, m, t_{j}\right)-\hat{\lambda}_{e}\left(g, t_{j}\right)\right\}^{2}\right]
\end{aligned}
$$

with $\hat{\lambda}_{c}\left(g, t_{j}\right)=\hat{\Lambda}_{g 1, c}+\hat{\Lambda}_{g 2, c}\left(t_{j}-t_{c}\right)$ and $\hat{\lambda}_{e}\left(g, t_{j}\right)=\hat{\Lambda}_{g 1, e}+\hat{\Lambda}_{g 2, e}\left(t_{j}-t_{e}\right)$ where $\hat{\Lambda}_{g 1, c}, \hat{\Lambda}_{g 2, c}, \hat{\Lambda}_{g 1, e}$ and $\hat{\Lambda}_{g 2, e}$ are the regression coefficients of the two linear models in Eq.B.1.

When all GCMs have the same number of runs $(M)$, the expression of model uncertainty in Eq.B.2 reduces to

$$
\widehat{s_{\alpha}^{2}}\left(t_{e}\right)=s_{\widehat{\alpha}}^{2}\left(t_{e}\right)-\frac{1}{M T^{*}} \hat{\sigma}_{\eta}^{2}
$$

where $\widehat{\sigma}_{\eta}^{2}$ is an unbiased estimator of internal variability variance for $X$

$$
\widehat{\sigma}_{\eta}^{2}=\frac{2}{G} \sum_{g=1}^{G} \widehat{\sigma_{\nu_{g}}^{2}}
$$

\section{Appendix C Simulation of MMEs}

Each MME is simulated for $Y(g, m, t)$ assuming that, for all $t_{s} \leq t \leq t_{f}$ :

$$
Y(g, m, t)=\lambda(g, t)+\nu(g, m, t),
$$


where the climate response function $\lambda(g, t)$ is a linear function of time 725 and where the $\nu(g, m, t)$ are independent and homoscedastic random variables (with variance $\left.\sigma_{\nu_{g}}^{2}\right)$. For convenience we further assume that $\lambda(g, t)$ can be decomposed as $\lambda(g, t)=w(t)+d(g, t)$, for $t=t_{1}, \ldots, T$ where the mean climate response $w(t)$ of the $G$ chains and the deviations $d(g, t)$ of chain $g$ are linear functions of time, expressed as: $w(t)=B+P .\left(t-t_{1}\right) /\left(t_{e}-t_{C}\right)$ and $d(g, t)=D(g) \cdot\left(t-t_{1}\right) /\left(t_{e}-t_{C}\right)$ with the constraint $\sum_{g=1}^{G} D(g)=0$.

For graphical simplification purposes, each MME for $Y$ is constructed so that the parameters $\left(\sigma_{\nu}^{2}, P, D(g), g=1, \ldots, G\right)$ lead, for the change variable $X$ at time $t=t_{e}$, to a prescribed value of the Response-to-Uncertainty ratio $\left[\mathrm{R} 2 \mathrm{U}\left(t_{e}\right)\right]$ and to a prescribed value of the fractional variance $F_{\eta}\left(t_{e}\right)$ due to internal variability.

For $X$, we have by definition $\varphi(g, t)=\lambda(g, t)-\lambda\left(g, t_{C}\right)$ and $\eta(g, m, t)=$ $\nu(g, m, t)-\nu\left(g, m, t_{C}\right)$. We have thus for the change variable $\mu(t)=w(t)-$ $w\left(t_{C}\right), \alpha(g, t)=d(g, t)-d\left(g, t_{C}\right)$ and in turn $\mu(t)=P .\left(t-t_{C}\right) /\left(t_{e}-t_{C}\right)$ and $\alpha(g, t)=D(g) .\left(t-t_{C}\right) /\left(t_{e}-t_{C}\right)$.

The theoretical values for $\mu\left(t_{e}\right)$ and $s_{\alpha}^{2}\left(t_{e}\right)$ are thus as follows $: \mu\left(t_{e}\right)=P$ and $s_{\alpha}^{2}\left(t_{e}\right)=\operatorname{Var}(D(g))$. Fixing $P$ to 1 , we thus simply require in turn

$$
\begin{array}{r}
\sigma_{X}^{2}\left(t_{e}\right)=\frac{1}{\left[R 2 U\left(t_{e}\right)\right]^{2}} ; \\
\sigma_{\nu}^{2}\left(t_{e}\right)=\frac{1}{2} \sigma_{\eta}^{2}\left(t_{e}\right)=\frac{1}{2} F_{\eta}\left(t_{e}\right) \cdot \sigma_{X}^{2}\left(t_{e}\right) ; \\
\operatorname{Var}(D(g))=s_{\alpha}^{2}\left(t_{e}\right)=\left(1-F_{\eta}\left(t_{e}\right)\right) \cdot \sigma_{X}^{2}\left(t_{e}\right) .
\end{array}
$$

For each MME simulation, the deviations of the different chains, $D(g), g=$ $1, \ldots, G$, are obtained from a sample of $G$ realizations in a normal distribution. These realizations are scaled so that their mean is zero and their variance corresponds to the prescribed value $s_{\alpha}^{2}\left(t_{e}\right)$.

\section{References}

Bosshard T, Carambia M, Goergen K, Kotlarski S, Krahe P, Zappa M, Schaer C (2013) Quantifying uncertainty sources in an ensemble of hydrological climate-impact projections. Water Resources Research 49(3):1523-1536

Bracegirdle TJ, Turner J, Hosking JS, Phillips T (2014) Sources of uncertainty in projections of twenty-first century westerly wind changes over the Amundsen Sea, West Antarctica, in CMIP5 climate models. Climate Dynamics 43(7-8):2093-2104

Braun M, Caya D, Frigon A, Slivitzky M (2012) Internal variability of the Canadian RCMs hydrological variables at the basin scale in Quebec and Labrador. Journal of Hydrometeorology 13(2):443-462

Charlton-Perez AJ, Hawkins E, Eyring V, Cionni I, Bodeker GE, Kinnison DE, Akiyoshi H, Frith SM, Garcia R, Gettelman A, Lamarque JF, Nakamura T, Pawson S, Yamashita Y, Bekki S, Braesicke P, Chipperfield MP, 
Dhomse S, Marchand M, Mancini E, Morgenstern O, Pitari G, Plummer D, Pyle JA, Rozanov E, Scinocca J, Shibata K, Shepherd TG, Tian W, Waugh DW (2010) The potential to narrow uncertainty in projections of stratospheric ozone over the 21st century. Atmospheric Chemistry and Physics 10(19):9473-9486

Cleveland S W (1979) Robust locally weighted regression and smoothing scatterplots. Journal of the American Statistical Association 74(368):829-836

Deser C, Phillips A, Bourdette V, Teng H (2012) Uncertainty in climate change projections: the role of internal variability. Climate Dynamics 38(3-4):527546

Evin G, Eckert N, Hingray B, Verfaillie D, Morin S, Lafaysse M, JBlanchet (2018) Traiter l'incertitude des projections climatiques: l'exemple des conditions meteorologiques et d'enneigement dans les Alpes francaises. Schweizerische Zeitschrift fur Forstwesen 169(4):203-209

Gelman A (2005) Analysis of variancewhy it is more important than ever. Annals of Statistics 33(1):1-53

Giuntoli J, Vidal JP, Prudhomme C, Hannah DM (2015) Future hydrological extremes: the uncertainty from multiple global climate and global hydrological models. Earth Syst Dynam 16:267-285

Hartley HO, Rao JNK (1967) Maximum-likelihood estimation for the mixed analysis of variance model. Biometrika 54(1/2):93-108

Hawkins E, Sutton R (2009) The potential to narrow uncertainty in regional climate predictions. Bulletin of the American Meteorological Society 90(8):1095-1107

Hawkins E, Sutton R (2011) The potential to narrow uncertainty in projections of regional precipitation change. Climate Dynamics 37(1-2):407-418

Hingray B, Blanchet J (2018) Uncertainty components estimates in transient climate projections. bias of moment-based estimators in the single time and time series approaches. Tech. rep., IGE, Univ-Grenoble Alpes, Grenoble, FR, https://hal.archives-ouvertes.fr/hal-01738218

Hingray B, Saïd M (2014) Partitioning internal variability and model uncertainty components in a multimodel multimember ensemble of climate projections. Journal of Climate 27(17):6779-6798

Hingray B, Mezghani A, Buishand TA (2007) Development of probability distributions for regional climate change from uncertain global mean warming and an uncertain scaling relationship. Hydrology and Earth System Sciences 11(3):1097-1114

Jacob D, Petersen J, Eggert B, Alias A, Christensen OB, Bouwer LM, Braun A, Colette A, Deque M, Georgievski G, Georgopoulou E, Gobiet A, Menut L, Nikulin G, Haensler A, Hempelmann N, Jones C, Keuler K, Kovats S, Kroener N, Kotlarski S, Kriegsmann A, Martin E, van Meijgaard E, Moseley C, Pfeifer S, Preuschmann S, Radermacher C, Radtke K, Rechid D, Rounsevell M, Samuelsson P, Somot S, Soussana JF, Teichmann C, Valentini R, Vautard R, Weber B, Yiou P (2014) EURO-CORDEX: new high-resolution climate change projections for European impact research. Regional Environmental Change 14(2):563-578 
Johns T, Royer JF, Höschel I, Huebener H, Roeckner E, Manzini E, May W, Dufresne JL, Otteå O, Vuuren D, Salas y Melia D, Giorgetta M, Denvil S, Yang S, Fogli P, Körper J, Tjiputra J, Stehfest E, Hewitt C (2011) Climate change under aggressive mitigation: the ensembles multi-model experiment. Climate Dynamics 37(9-10):1975-2003

Kendon EJ, Rowell DP, Jones RG, Buonomo E (2008) Robustness of future changes in local precipitation extremes. Journal of Climate 21(17):42804297

Kew SF, Selten FM, Lenderink G, Hazeleger W (2011) Robust assessment of future changes in extreme precipitation over the Rhine basin using a GCM. Hydrology and Earth System Sciences 15(4):1157-1166

Lafaysse M, Hingray B, Mezghani A, Gailhard J, Terray L (2014) Internal variability and model uncertainty components in a multireplicate multimodel ensemble of hydrometeorological projections : the Alpine Durance basin. Water Resources Research 50:3317-3341

Montgomery DC (2012) Design and Analysis of Experiments., 8th edn. Wiley

Northrop PJ, Chandler RE (2014) Quantifying sources of uncertainty in projections of future climate. Journal of Climate 27(23):8793-8808

Olonscheck D, Notz D (2017) Consistently Estimating Internal Climate Variability from Climate Model Simulations. Journal of Climate 30(23):95559573, DOI $\{10.1175 /$ JCLI-D-16-0428.1 $\}$

Paeth H, Vogt G, Paxian A, Hertig E, Seubert S, Jacobeit J (2017) Quantifying the evidence of climate change in the light of uncertainty exemplified by the Mediterranean hot spot region. Global and Planetary Change 151(SI):144151, DOI \{10.1016/j.gloplacha.2016.03.003\}

van Pelt SC, Beersma JJ, Buishand TA, van den Hurk BJJM, Schellekens J (2015) Uncertainty in the future change of extreme precipitation over the Rhine basin: the role of internal climate variability. Climate Dynamics 44(78):1789-1800

Räisänen J (2001) CO2-induced climate change in CMIP2 experiments: Quantification of agreement and role of internal variability. Journal of Climate 14(9):2088-2104

Reintges A, Martin T, Latif M, Keenlyside NS (2017) Uncertainty in twentyfirst century projections of the Atlantic Meridional Overturning Circulation in CMIP3 and CMIP5 models. Climate Dynamics 49(5-6):1495-1511, DOI $\{10.1007 / \mathrm{s} 00382-016-3180-\mathrm{x}\}$

Vidal JP, Hingray B, Magand C, Sauquet E, Ducharne A (2016) Hierarchy of climate and hydrological uncertainties in transient low-flow projections. Hydrology and Earth System Sciences 20(9):3651-3672, DOI \{10.5194/hess-20-3651-2016\}

Yip S, Ferro CAT, Stephenson DB, Hawkins E (2011) A simple, coherent framework for partitioning uncertainty in climate predictions. Journal of Climate 24(17):4634-4643 


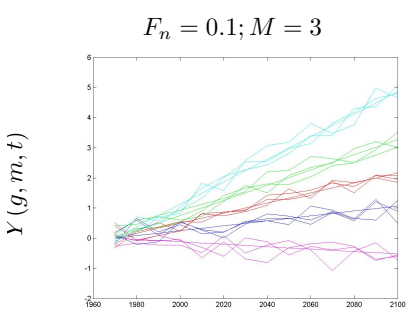

year

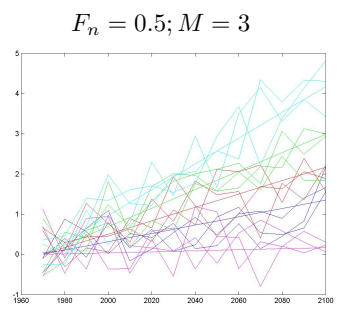

year

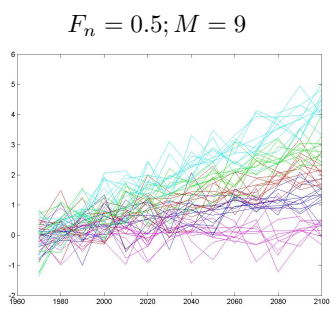

year

Fig. 1 Three synthetic multimodel multimember ensembles of time series projections (MMEs) obtained via simulation with different $F_{\eta}$ ratios and $M$ numbers (left: $F_{\eta}=0.1, M=3$; middle $F_{\eta}=0.5, M=3$; right: $F_{\eta}=0.5, M=9$ ). Time series over the 1960-2100 period for the raw projections $Y(g, m, t)$ of a 10 -yr average climate variable. Cases on the left and in the middle are illustrative of typical configurations encountered in climate analyses: "small" number of members available for each GCM $(M=3)$ with relatively low (resp. high) contribution of IV to total variance (case $1: F_{\eta}=0.1$; case $2: F_{\eta}=0.5$ ). The case on the right is similar as the middle case but in a configuration with a (unrealistic) "large" number of members $(M=9)$. 

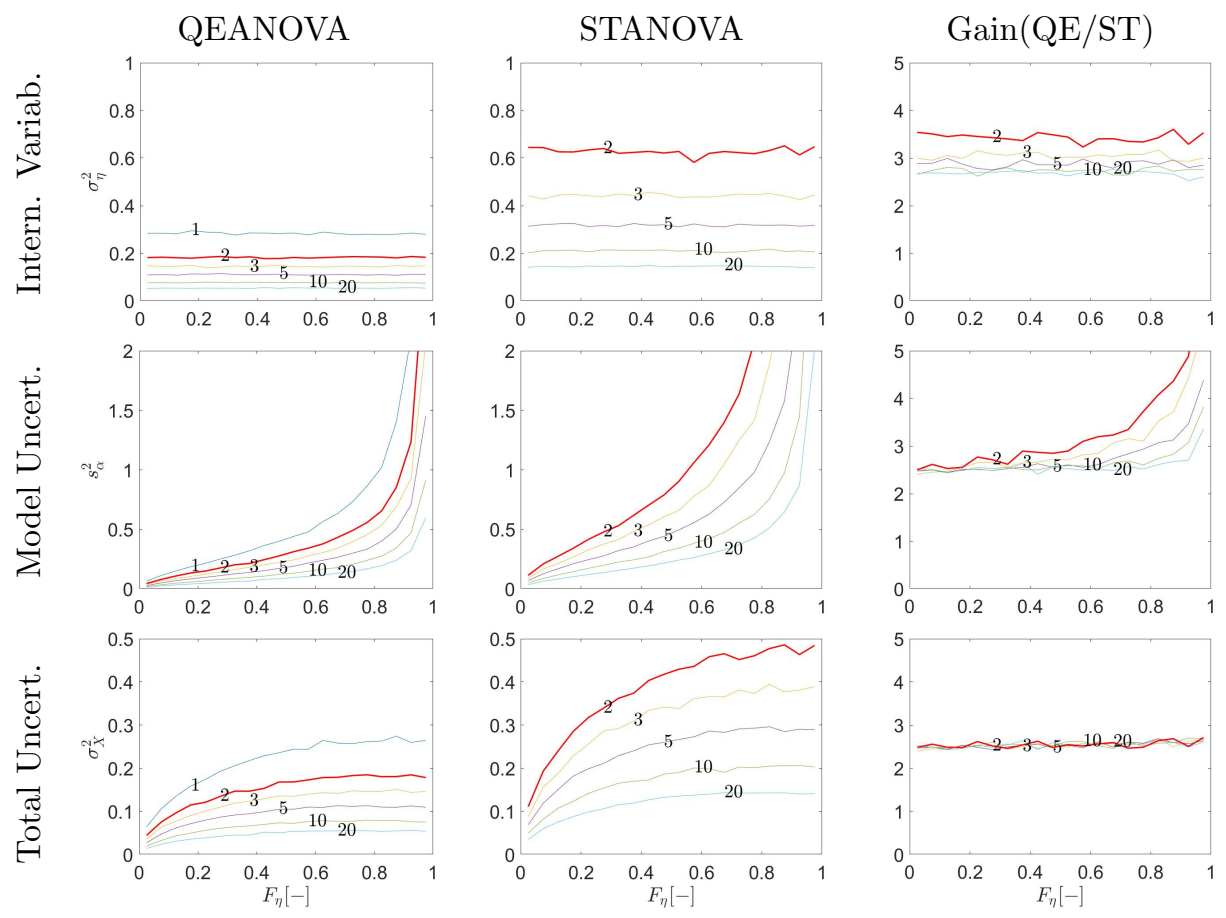

Fig. 2 Precision of QEANOVA (left) and STANOVA (middle) estimates for uncertainty variance components, and gain in precision between STANOVA SD values and QEANOVA SD values (right) (gain = ratio between STANOVA SD values and QEANOVA SD values). Top: Internal Variability, middle: Model Uncertainty, bottom: Total Uncertainty. SD values are given as a function of the fraction of total variance explained by internal variability $\left(F_{\eta}\right)$ for a few representative values of number of members $M$ : $(1), 2,3,5,10,20$. Results are presented for a theoretical ratio $R 2 U=1$. For the sake of clarity, the upper limit of the figures for $s_{\alpha}^{2}$ is truncated to 2 . The highest values are greater than 10. Figures of the ratios between SD values obtained with both approaches are truncated to 5 . 

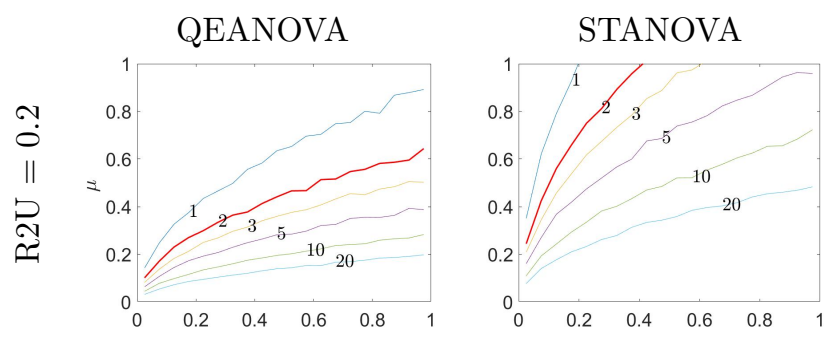

\section{Gain(QE/ST)}
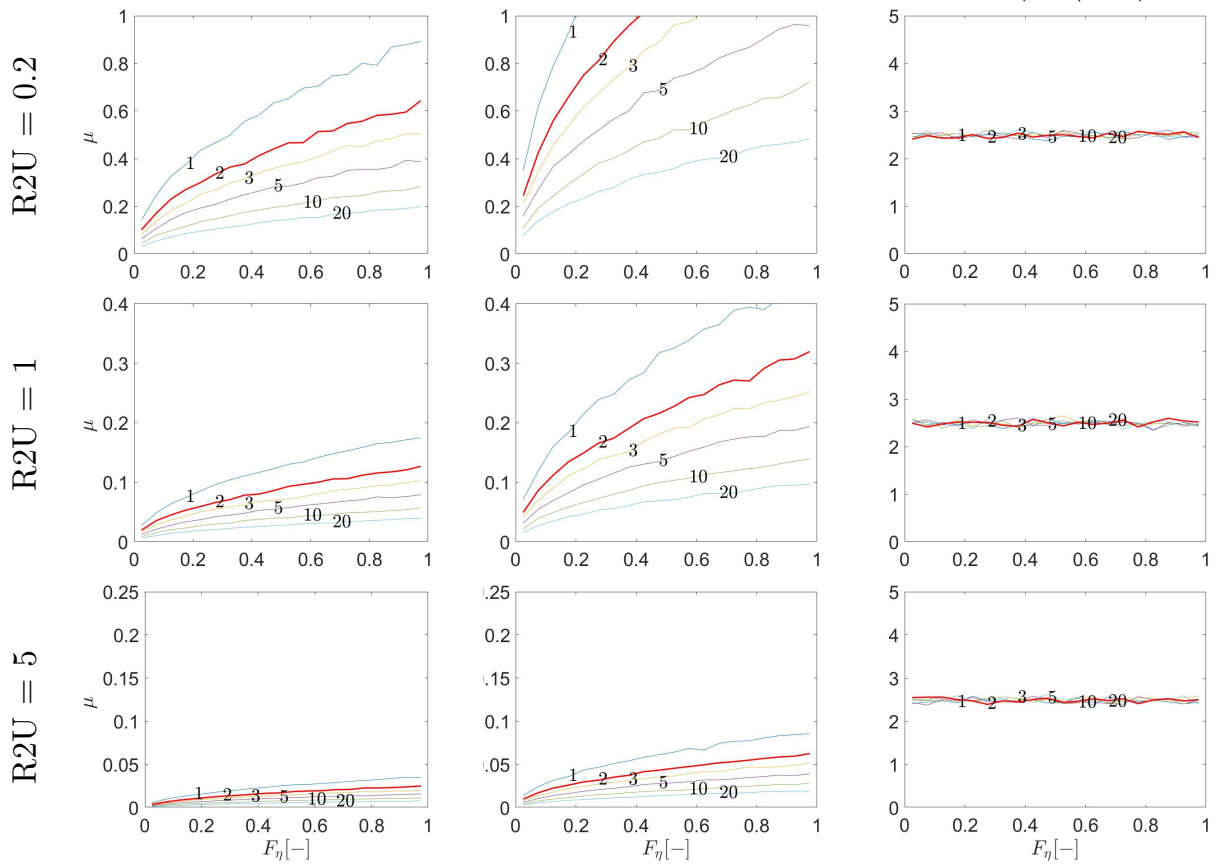

Fig. 3 Precision of QEANOVA (left) and STANOVA (middle) estimates for mean change response estimates $(\mu)$. Gain in precision between STANOVA and QEANOVA in the right panels. Results are presented for different values of the theoretical response-to-uncertainty ratio $\mathrm{R} 2 \mathrm{U}$ (top: $R 2 U=0.2$, middle: $R 2 U=1$, bottom: $R 2 U=5$ ). For details see caption of Figure 2. 

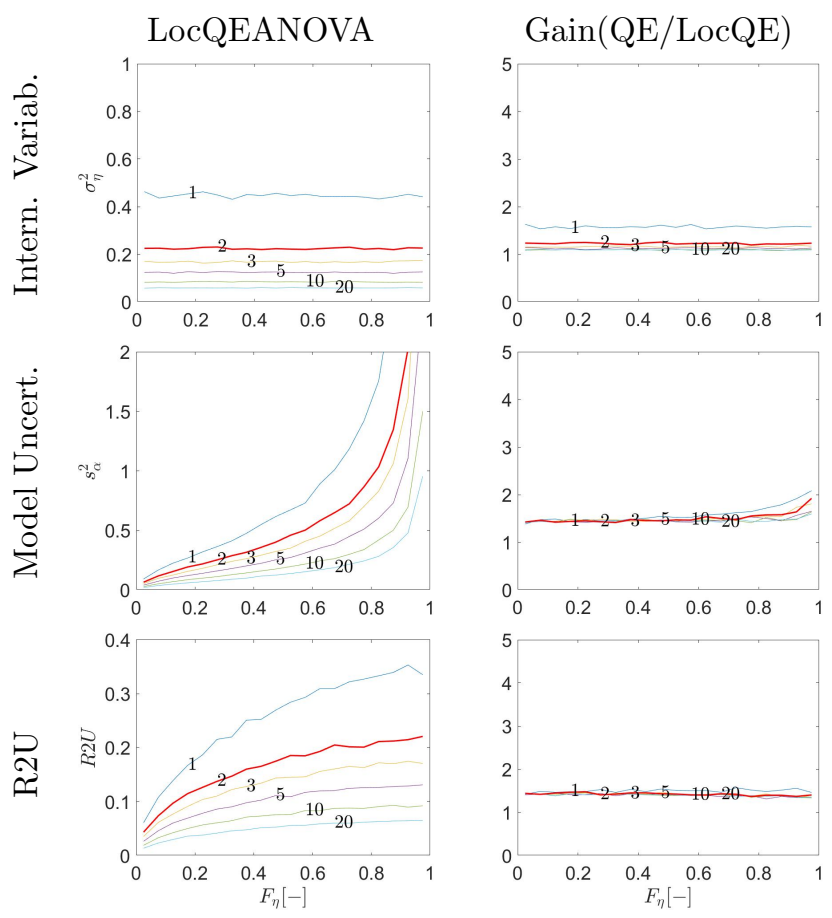

Gain(LocQE/ST)
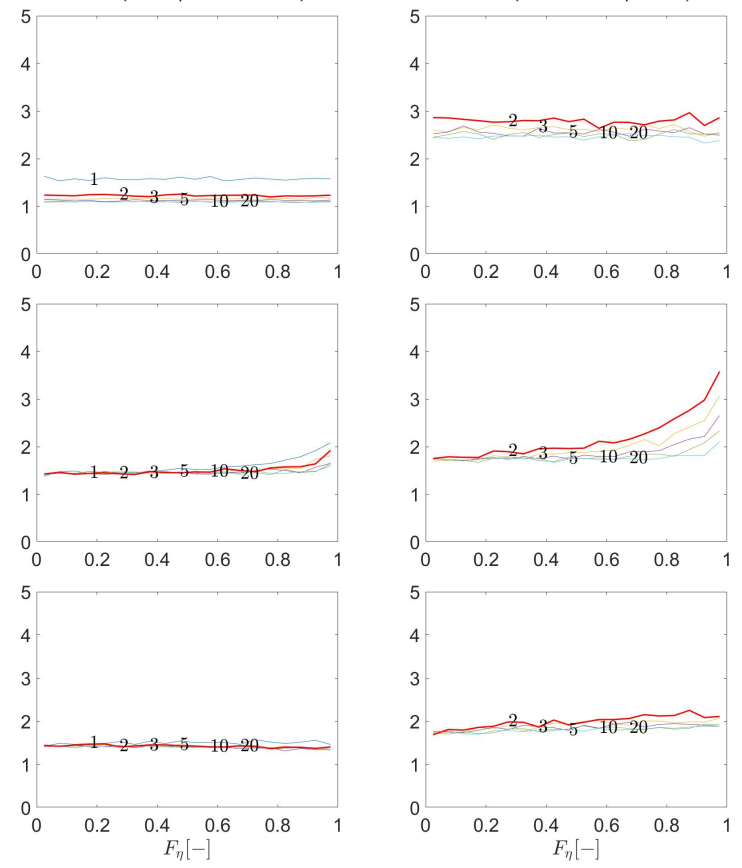

Fig. 4 Precision of uncertainty estimates obtained with the local QEANOVA approach. Results for internal variability variance $\left(\sigma_{\eta}^{2}\right.$, top), model uncertainty variance $\left(s_{\alpha}^{2}\right.$, middle), response-to-uncertainty ratio (R2U, bottom). Left: SD values, middle: gain in precision between local QEANOVA and QEANOVA approaches (ratio between local QEANOVA SD values and QEANOVA SD values), right: gain in precision between STANOVA and local QEANOVA approaches (ratio between STANOVA SD values and local QEANOVA SD values). Results for a theoretical ratio $R 2 U=1$. For details see caption of Figure 2 . 
STANOVA
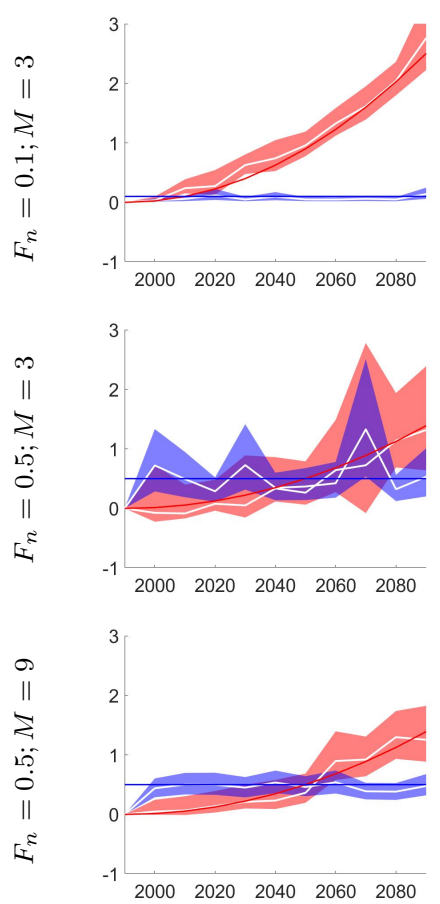

LocalQEANOVA
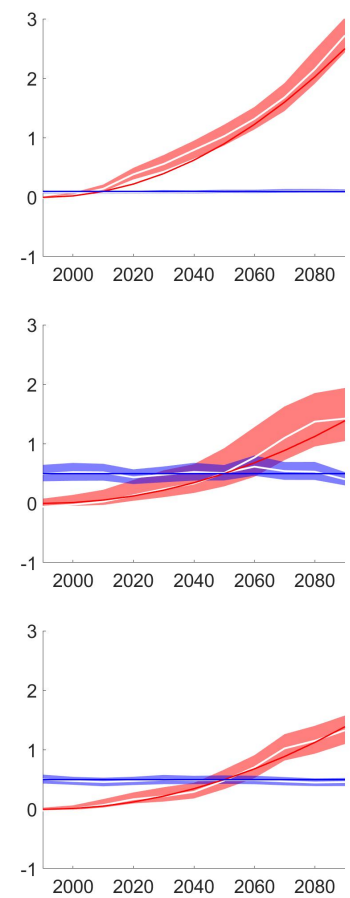

QEANOVA
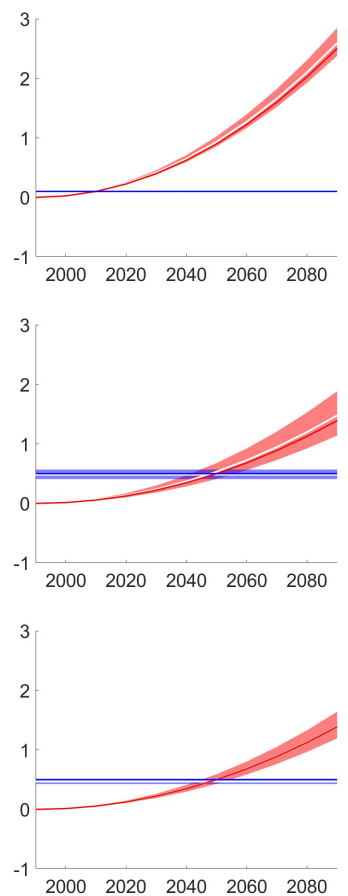

Fig. 5 Temporal variations and $\mathbf{9 0 \%}$ confidence intervals of model uncertainty and internal variability estimates for the three reference datasets presented in Fig. 1 (top: $F_{\eta}=0.1, M=3$; middle $F_{\eta}=0.5, M=3$; bottom: $F_{\eta}=0.5, M=9$ ). Left: with STANOVA; middle: with local QEANOVA where $\Omega(t)$ is composed of the 3 consecutive time steps centered around $t$; right: with QEANOVA. $s_{\alpha}^{2}$ in red, $\sigma_{\eta}^{2}$ in blue. The blue and red lines correspond to the theoretical variance components used for the generation of the reference dataset. The variance component estimates (white curves) obtained with each ANOVA approach are presented with their $90 \%$ confidence intervals. 

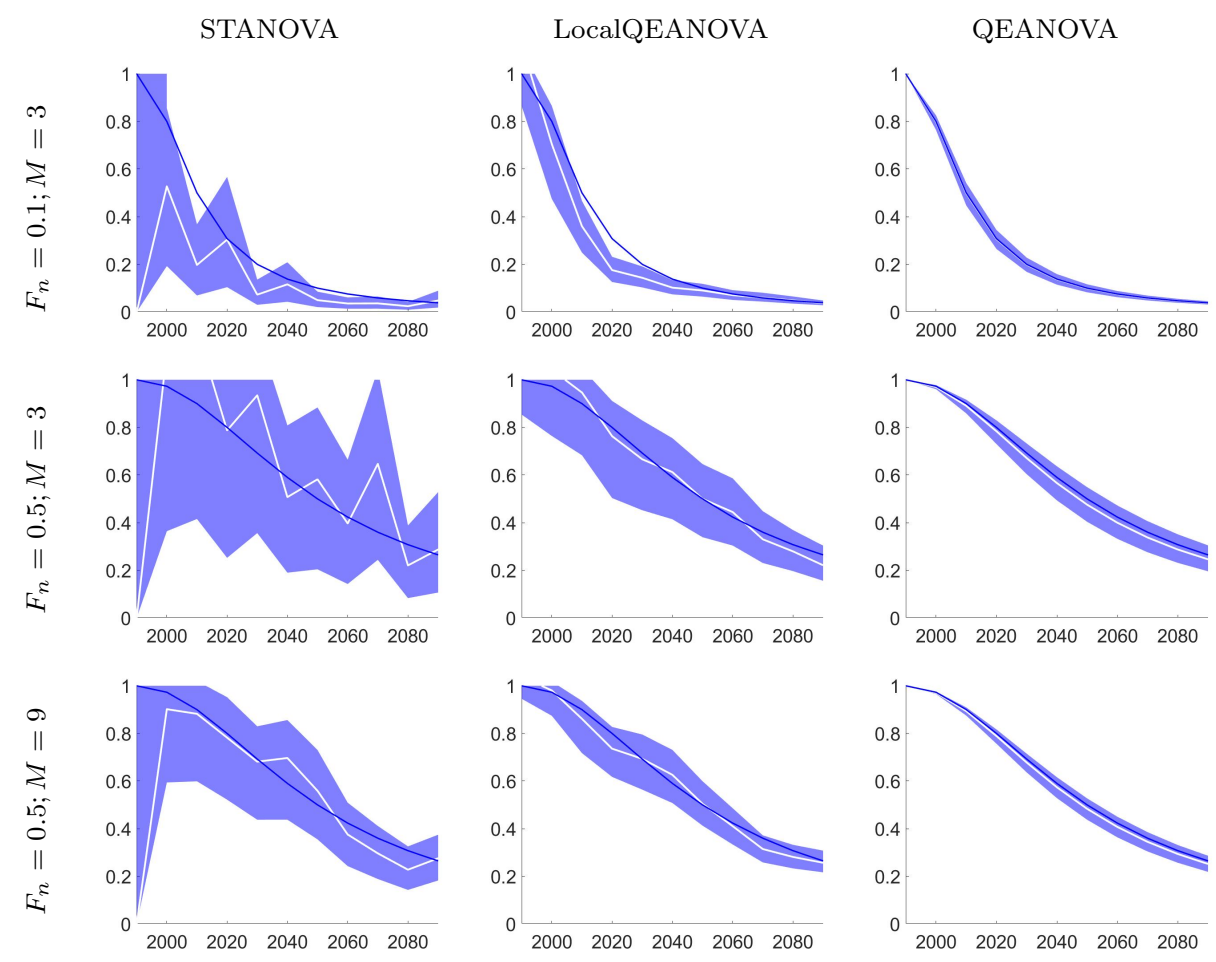

Fig. 6 Temporal variations and $90 \%$ confidence intervals of uncertainty estimates for the fractional variance due to internal variability $\left(F_{\eta}\right.$ ratio) for the three reference datasets presented in Fig. 1. Left: with STANOVA; middle: with local QEANOVA right: with QEANOVA. The blue lines correspond to the theoretical fractional variance used for the generation of the reference dataset. See Fig. 5 for caption details. 